\title{
AGING AND THE EFFECTS OF CONVERSATION WITH A PASSENGER OR A CALLER ON SIMULATED DRIVING PERFORMANCE
}

\author{
Ensar Becic, Tate Kubose, Arthur F. Kramer, \\ Gary S. Dell, Susan M. Garnsey, \& Kathryn Bock \\ Beckman Institute and Department of Psychology \\ University of Illinois \\ Urbana-Champaign, IL, United States \\ E-mail: ebecic@uiuc.edu
}

\begin{abstract}
Summary: A total of 96 pairs of older and younger drivers participated in a study assessing the effects of conversation on the driving performance of older and younger drivers in a simulated city environment. These effects were investigated while drivers conversed with an in-vehicle passenger or an outside-vehicle caller. All of the passengers completed three separate, counterbalanced blocks of tasks that consisted of two single-task blocks (driving only and conversing only) and one dual-task block (driving and conversing). The results showed greater variability in velocity, lane keeping and steering control under single-task than under dual-task conditions. Drivers also showed greater average velocity and greater deviation from the center of the lane under single-task than under dualtask conditions. However, when crossing an intersection, a task requiring greater attentional resources, drivers exhibited a cost due to the dual task. Our data are consistent with the literature, which suggests that a secondary task may aid in the performance of a routinized task but may also impose costs if the primary task requires significant attentional resources. Older drivers exhibited greater variability in velocity, stayed closer to the center of the lane, and waited longer to cross intersections than their younger counterparts, suggesting that they compensate for their declining perceptual and cognitive abilities through changes in driving behavior. Drivers exhibited greater variability in steering under singletask conditions when talking to an outside-vehicle caller, and older drivers showed greater variability in velocity when conversing with an outside-vehicle caller.
\end{abstract}

\section{Introduction}

According to recent studies, $85 \%$ of cell phone users in the United States talk on their cell phones when driving (Goodman, Tijerina, Bents \& Wierwille, 1999), and those drivers are four times more likely to be involved in an accident (Redelmeir \& Tibsihirani, 1997; LaBergeNadeau et al., 2003). These facts have stimulated a great deal of research and even some legislative action, such as New York City's 2001 ban of hand-held cell phones while driving (Chapter 69 of the Laws of 2001, section 1225c State of New York), while permitting hands-free use.

The physical manipulation of cell phones requires visuo-motor resources, so it is not surprising that previous studies have shown a negative impact of such manipulation on driving performance (Briem \& Hedman, 1995). However, the manual manipulation (dialing, answering) is only a 
minor component of the distraction from cell-phone conversation. Strayer and Johnston (2001) conducted a simulated driving study in which participants performed a driving task while talking to a confederate using either a hand-held or hands-free cell phone. Drivers missed more traffic signs and responded more slowly to events when talking to another person, but these dual-task deficits were the same for hands-free and hand-held cell phones. Similar findings have been shown in a study by Lamble, Kauranen, Laasko and Summala (1999).

Difficulty in dual-task situations is one of the hallmarks of declines related to aging, so it might be expected that driving performance while conversing on a cell phone would decline in older adults. In a lead-vehicle following task, Strayer and Drews (2004) found slower response times to the random braking of a lead vehicle and a greater following distance when drivers were engaged in a cell-phone conversation with another person compared to when they were just driving. More importantly, these differences were equivalent for older and younger adults, suggesting that older drivers don't experience an additional cost in driving performance when engaged in a conversation. Kramer, Cassavaugh, Horrey, Becic and Mayhugh (in press), who also failed to find aging differences in response times to unexpected events, attributed it to the much greater driving experience of older adults.

Most of the driving studies investigating the effects of conversation on driving performance use a conversation task in which a participant talks to a confederate about an already predetermined topic. While this approach has the virtue of naturalness, it suffers from a lack of control over the conversation.

In the current study we use a story-retelling task (Lockridge \& Brennan, 2002) to assess the effects of conversation on the driving performance of older and younger drivers in a simulated city environment. These factors are explored while the driver converses either with an in-vehicle passenger or an outside-vehicle caller. By using a controlled speech task, we hope to determine the effects of conversation with a passenger as opposed to with someone outside the vehicle (simulating a hands-free cell phone conversation) on driving performance. Despite the research described above, there is strong evidence that older adults show deficits in multi-task performance. Therefore, we decided to examine potential age differences in conversation effects on simulated driving performance.

\section{Methods}

Participants. A total of 96 pairs of adults participated in the study. Each pair consisted of a driver and a passenger/caller. Half of the participant pairs were older (ranging from 57-88 with an average of 71.1 years) and half were younger (ranging from 18-24 with an average of 19.6 years) adults. All of the participants had normal or corrected to normal vision.

Driving Simulator. This study was completed in the Beckman Driving Simulator at the University of Illinois (GlobalSim, Inc. Vection ${ }^{\mathrm{TM}}$ Research Simulator). This is a fixed-base simulator consisting of a 1998 Saturn SL with a $135^{\circ}$ wraparound forward and $135^{\circ}$ rear fields. Six Epson PowerLite 703c projectors project the simulated environment on separate screens (three in front and three in the rear). The simulator dynamics and environment were managed by 
GlobalSim's Vection ${ }^{\mathrm{TM}}$ software version 1.6.1. All participants were equipped with headphones and microphones.

Driving scenarios and environments were created with GlobalSim's HyperDrive ${ }^{\mathrm{TM}}$ authoring software version 1.6.1. Intersection and ambient traffic was created through scripts.

Procedure. Half the participants in each age group were assigned to an in-vehicle condition (passenger in the car) while the other half were assigned to an outside-vehicle condition (caller in a sound-proof booth, outside the simulator). All of the passengers completed three separate blocks of tasks that were counterbalanced and consisted of two single-task blocks (driving only and conversing only) and one dual-task block (driving and conversing).

The speech task consisted of a story-retelling task (Lockridge \& Brennan, 2002). Each member of a pair, in turn, heard a story through headphones and was instructed to retell the story to their partner. A prompt announced that the driver/passenger was about to hear a story and to get ready. Then, only the announced member of the pair would hear a story through their headphones and was then asked to retell the story to their partner. The stories lasted 15 seconds and participants had 30 seconds to relay the details of the story to their partner. Then, another prompt announced that the other member of the pair was about to hear a story and the procedure was repeated with the roles of reteller and listener switched. Each member of the pair heard and was asked to retell four stories during the speech task. When performing the task, participants were instructed to listen to the stories carefully and relay them in a manner that would allow their partner to remember them. At the end of each block in which participants conversed, both members of a pair were asked to recall the stories they were told by their partners.

The driving task consisted of driving through an urban environment and crossing several busy intersections. The driver's lane was empty of other vehicles, and other than the intersections, the scenarios did not contain events that would require drivers to respond to a potential collision. When performing the driving task, participants were instructed to obey the $30 \mathrm{~m} / \mathrm{h}$ speed limit, stay in the center of their lane, stop at stop signs, and cross intersections in a safe and timely manner.

Design and Measures. Driving performance was measured by the mean and standard deviation of velocity, lane keeping, steering control and braking, as well as the time spent crossing the intersections. These measures were analyzed in a $2 \times 2 \times 2$ mixed design matrix with Task (dual or single) as a within-subjects measure and Age (older and younger) and Location of conversation partner (in-vehicle or outside-vehicle) as between-subject measures. Male and female drivers and passengers/callers were counterbalanced across conditions.

\section{Results and Discussion}

For the purpose of analysis, driving environment was divided into intersection and straightaway portions. The intersection part included 100 meters before an intersection to $100 \mathrm{~m}$ after the end of an intersection. Unless stated otherwise, driving performance measures are analyzed on straightaway portions of the driving environment. Also, driving performance measures in the 
dual-task condition include only those sections of the route during which participants were involved in a dual-task (either listening or retelling the stories).

A mixed mode ANOVA with Task (dual and single) as a within-subjects measure and Age and Location of the conversation partner as between-subjects measures was performed on the variability of lane position (see Figure 1). The results showed a main effect of Task $(\mathrm{F}(1,92)=$ $187.86, \mathrm{p}<.001)$. Drivers exhibited greater variability in lane position under single-task than under dual-task conditions. The main effect of task was also found for the variability of velocity $(\mathrm{F}(1,92)=9.61, \mathrm{p}=.003)$ and the variability in steering $(\mathrm{F}(1,92)=480.03, \mathrm{p}<.001)$ measures revealing greater variability under single-task conditions. Participants also stayed closer to the center of the lane $(F(1,92)=6.48, p=.013)$ and drove more slowly $(F(1,92)=22.72, p<.001)$ under dual-task than under single-task conditions. The findings of less greater variability in lane position keeping, steering and velocity under single-task than under dual-task conditions are in line with Kubose et al. (2006), who also found better lane control under dual-task conditions (driving and conversing). This may be due to a strategic compensatory response to additional tasks demands. An alternative explanation may be that distraction from conversation aids in the performance of routinized tasks, such as driving, by taking attention away from the performance, which is detrimental for such skills (e.g., Beilock, Afremow, Rabe \& Carr, 2001).

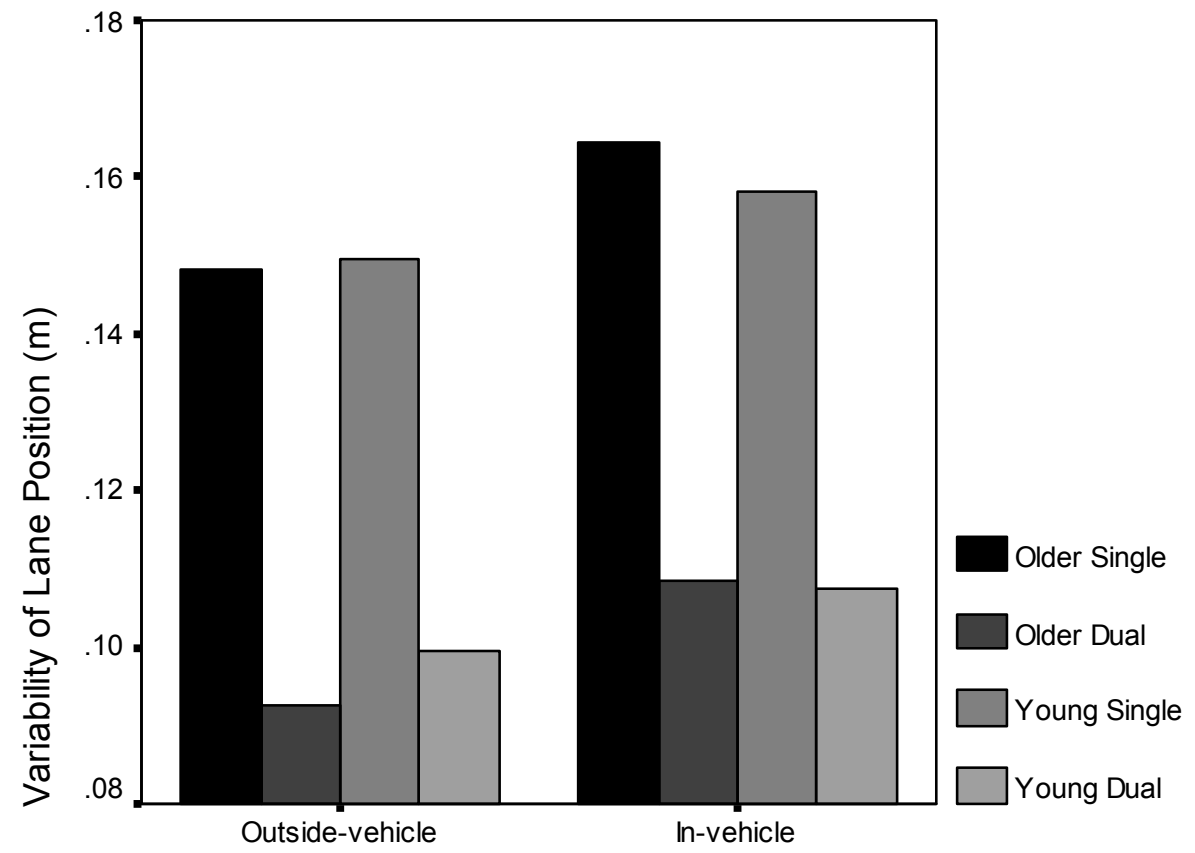

Location of the non-driver

Figure 1. Variability in lane position in meters as a function of age, task and location of conversation partner

Our analyses showed several ways in which older and younger drivers differ. Older drivers exhibited greater variability in velocity $(\mathrm{F}(1,92)=17.94, \mathrm{p}<.001)$ and stayed closer to the center of the lane $(\mathrm{F}(1,92)=4.32, \mathrm{p}=.040)$. A significant interaction between Age and Task for variability of velocity $(\mathrm{F}(1,92)=16.51, \mathrm{p}<.001)$ consisted in older adults exhibiting greater 
variability under single-task conditions compared to younger drivers (see Figure 2). This agerelated difference was not present under dual-task conditions. Older drivers matched the velocity of younger drivers on the straightaway sections, but they approached intersections with greater caution. This was exhibited in slower velocity $(F(1,92)=62.37, p<.001)$ and longer times to cross intersections $(\mathrm{F}(1,92)=61.79, \mathrm{p}<.001)$. Experience may moderate the age-related decline in cognitive and perceptual processes and enable aging drivers to prepare themselves for potential collisions (Kramer et al., in press), but it is clear that older drivers also modify their driving behavior to compensate for their declining perceptual processes.

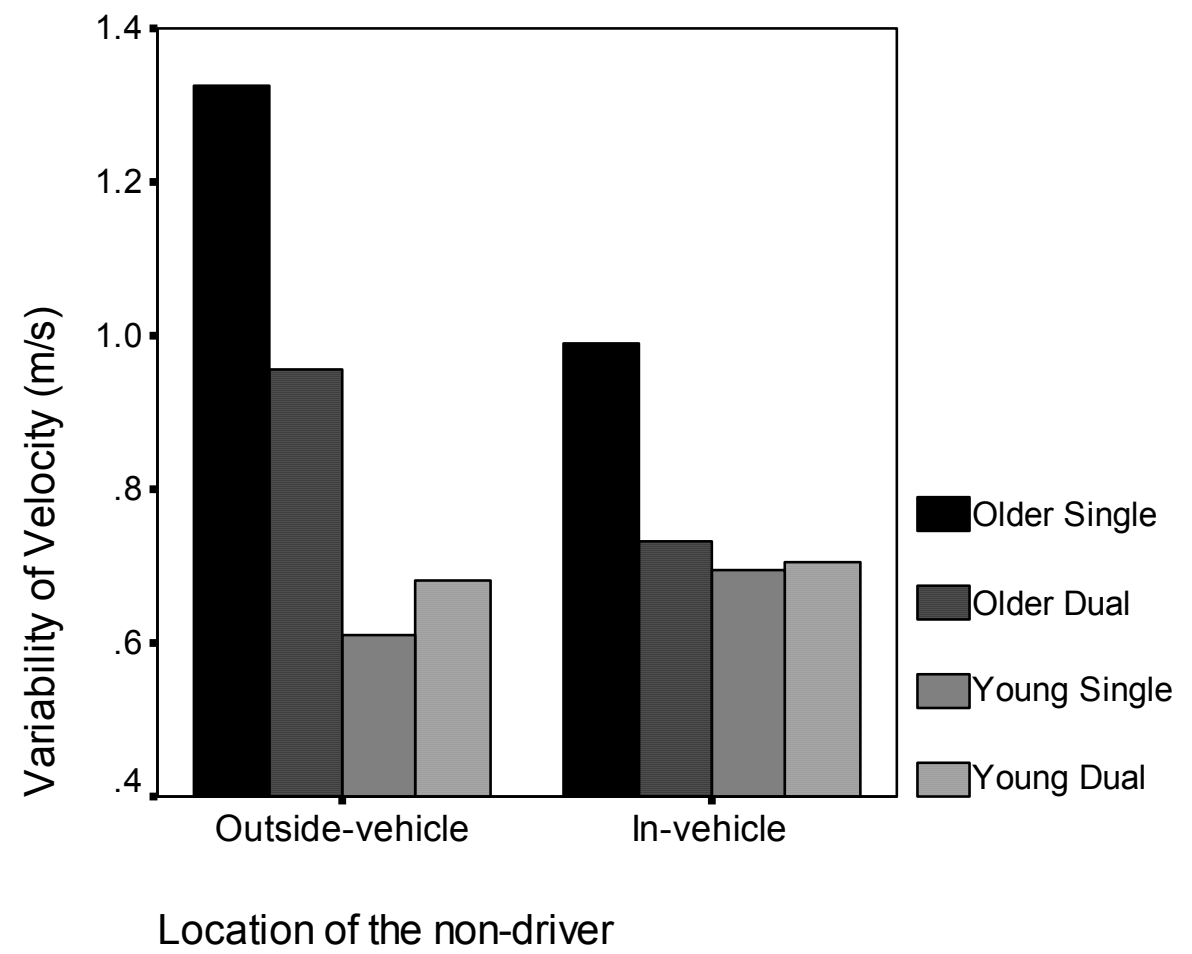

Figure 2. Variability in velocity (meters/second) as a function of age, task and location of conversation partner

The analyses also showed several interesting effects involving the location of the conversation partner. There was a significant interaction between Age and Location of the conversation partner for the velocity variability measure $(F(1,92)=4.67, p=.033)$. Older drivers showed greater variability in velocity in the outside-vehicle conversation condition than younger drivers $(\mathrm{p}=.001)$, but not in the in-vehicle conversation condition. An interaction between Task and Location of the conversation partner was also found for the steering variability measure $(\mathrm{F}(1,92)$ $=3.95 . \mathrm{p}=.05)$. Drivers exhibited greater variability in steering when conversing with an outside-vehicle caller compared to an in-vehicle passenger under dual-task conditions $(\mathrm{p}=.05)$, but this difference was not present under single-task conditions. Thus, it is important to take the driving environment context into account when interpreting the results of driving performance. While most aspects of driving can be considered a routinized task, crossing an intersection is a task that requires significant attentional resources. As such, dual-task processing is more likely to have a negative impact on the performance of this task and age-related differences may become 
more prominent. Drivers under dual-task conditions required more time crossing intersections, although this difference was not significant $(\mathrm{p}=.18)$. This trend was prominent in the outsidevehicle condition (see Figure 3), as suggested by a marginal interaction of Task and Location of the conversation partner $(p=.10)$.

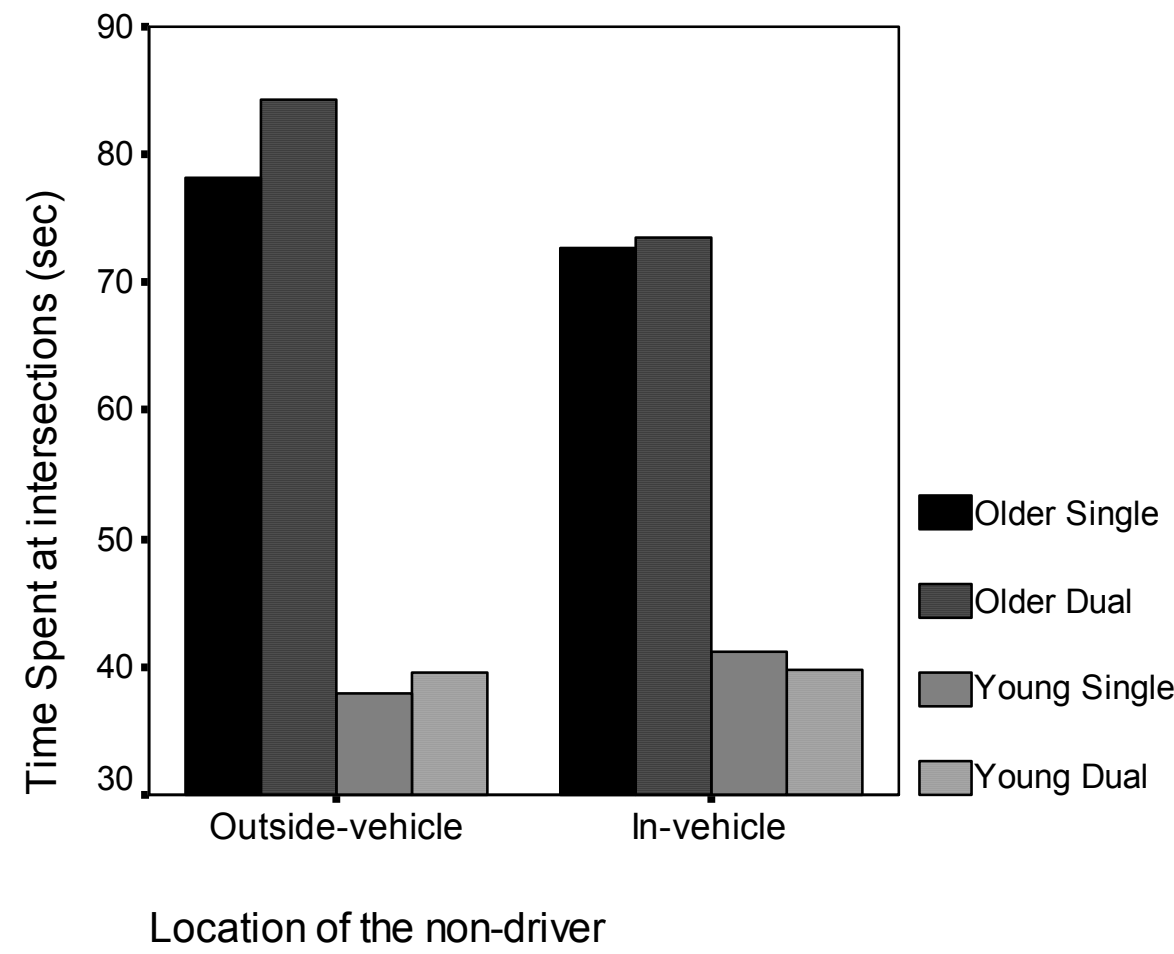

Figure 3. Time spent at intersection (seconds) as a function of age, task and the location of the conversation partner

Driving performance while conversing showed less variability in several driving performance measures, producing better lane position keeping and smaller velocity variability than driving without conversing. The ostensibly better driving performance under dual-task conditions needs to be interpreted within the context of driving environment. Better driving control was obtained on the section of environment that did not require drivers to respond in any manner (by braking or steering) to avoid potential collisions. However, when crossing an intersection, a task that requires greater attentional resources, drivers exhibited a cost when conversing. This may give further credence to an explanation proposed by Beilock et al. (2001), who suggested that secondary task aids in the performance of a routinized task. It is also possible that greater variability under single-task conditions is due to greater responsiveness to stimuli in the urban environment.Studies that explore this issue by systematically varying the number of external events that must be avoided should be considered in the future. Older drivers exhibited less velocity control and greater caution when approaching and crossing intersections. This compensatory driving behavior, along with driving experience, may be the factors that modulate age-related decline in perceptual processes. Additional analyses, completed over the next couple of months, will examine the relative impact of speaking versus listening on driving performance and the tradeoffs between conversing and driving under different experimental conditions. 


\section{REFERENCES}

Beilock, S., Afremow, J., Rabe, A. \& Carr, T. (2001). Don't miss! The debilitating effects of suppressive imagery on golf putting performance. Journal of Sport \& Exercise Psychology, 23, 200-221.

Briem, V., \& Hedman, L.R. (1995). Behavioural effects of mobile telephone use during simulated driving. Ergonomics, 38, 2536-2562.

Goodman, M.F., Tijerina, L., Bents, F.D., \& Wierwille, W.W. (1999). Using cellular telephones in vehicle: Safe or unsafe? Transportation Human Factors, 1, 3-42.

Kramer, A.F., Cassavaugh, N., Horrey, W.J., Becic, E., \& Mayhugh, J. (in press). Influence of age and proximity warning devices on collision avoidance in simulated driving. Human Factors.

Kubose, T., Bock, K., Dell, G.S., Garnsey, S.M., Kramer, A.F., Mayhugh, J. (2006). The effects of speech production and speech comprehension on simulated driving performance. Applied Cognitive Psychology, 1, 43-63.

Laberge-Nadeau, C., Maag, U., Bellavance, F., Lapierre, S., Desjardins, D., Messier, S., \& Saïdi, A. (2003). Wireless telephones and the risk of road crashes. Accident Analysis and Prevention, 35, 649-660.

Lamble, D., Kauranen, T., Laasko, M., \& Summala, H. (1999). Cognitive load and detection thresholds in car following situations: safety implications for using mobile (cellular) telephones while driving. Accident Analysis and Prevention, 31, 617-623.

Lockridge, C.B., \& Brennan, S.E. (2002). Addressees' needs influence speakers' early syntactic choices. Psychonomic Bulletin \& Review, 9, 550-557.

Redelmeir, D.A., \& Tibsihirani, R.J. (1997). Association between cellular-telephone calls and motor vehicle collisions. The New England Journal of Medicine, 336, 453-458.

Strayer, D.L., \& Drews, F.A. (2004). Profiles in driver distraction: Effects of cell phone conversation on younger and older drivers. Human Factors, 46, 640-649.

Strayer, D.L., \& Johnston, W.A. (2001). Driven to distraction: Dual-task studies of simulated driving and conversing on a cellular phone. Psychological Science, 12, 462-466. 